\title{
Endothelial function evaluation in patients with anorexia
} \section{nervosa}

\author{
Patcharapong Suntharos* ${ }^{* 1,2}$, Myriam E Almeida Jones ${ }^{1,3}$, Howard S Seiden ${ }^{1,4}$, Martin Fisher ${ }^{5}$, Dorota Gruber ${ }^{1}$, Lisa M Rosen ${ }^{6}$, Andrew D \\ Blaufox ${ }^{1}$ and Rubin S. Cooper ${ }^{1}$ \\ ${ }^{1}$ The Division of Pediatric Cardiology, Department of Pediatrics, Cohen Children's Medical Center of New York, Northwell Health, NY, USA \\ ${ }^{2}$ The Department of Pediatric Cardiology, Cleveland Clinic Children's Hospital, OH, USA \\ ${ }^{3}$ The Division of Cardiology, Department of Pediatrics, University of California Irvine and Children's Hospital of Orange County, CA, USA \\ ${ }^{4}$ The Division of Pediatric Cardiology, Kravis Children's Hospital at Mount Sinai, NY, USA \\ ${ }^{5}$ The Division of Adolescent Medicine, Department of Pediatrics, Cohen Children's Medical Center of New York, Northwell Health, NY, USA \\ ${ }^{6}$ Biostatistics Unit, The Feinstein Institute for Medical Research, Northwell Health, NY, USA
}

\begin{abstract}
Introduction: This study evaluated endothelial function in patients with anorexia nervosa (AN) using Endothelial Pulse Amplitude Testing (Endo-PAT) and correlated findings with the patients' history and biochemical data.

Method: Twenty-one patients age 13-21 years diagnosed with AN by the Division of Adolescent Medicine at Cohen Children's Medical Center of New York between 6/1/2012 and 5/31/2013 were studied along with 19 healthy controls similar in age and gender distribution. Digital pulse amplitude was examined using Endo-PAT. Raw data were automatically transferred into a reactive hyperemia index (RHI) and the natural log transformation of RHI (LnRHI). Subjects' and controls' electrocardiograms and biochemical markers were obtained.

Results: AN and controls had similar RHI $(\mathrm{P}=0.7542)$ and LnRHI $(\mathrm{P}=0.9497)$. AN had lower mean weight $(\mathrm{P}<0.0001)$, height $(\mathrm{P}=0.0207)$, BMI $(\mathrm{P}<0.0001)$, resting HR $(\mathrm{P}<0.0001)$, systolic $(\mathrm{P}<0.0001)$ and diastolic $\mathrm{BP}(\mathrm{P}=0.0141)$. AN also had lower mean HR during EndoPAT testing $(\mathrm{P}<0.0001)$, triiodothyronine $(\mathrm{T} 3)$ $(\mathrm{P}<0.0001)$, luteinizing hormone $(\mathrm{LH})(\mathrm{P}=0.0055)$ and estradiol $\left(\mathrm{E}_{2}\right)(\mathrm{P}=0.0052)$. Total cholesterol $(\mathrm{Chol})(\mathrm{P}=0.0004)$ was higher in $\mathrm{AN}$ subjects. No correlation was observed between $\mathrm{RHI}$ and other parameters.
\end{abstract}

Conclusion: No significant differences in RHI or LnRHI were found between the two groups. There were significantly higher Chol and lower $\mathrm{HR}, \mathrm{T} 3, \mathrm{LH}$ and $\mathrm{E}_{2}$ levels in the AN group compared to controls. There were no correlations of these parameters to RHI.

\begin{abstract}
Abbreviations: AN: anorexia nervosa; BMI: body mass index; Chol: total cholesterol; DBP: diastolic blood pressure; $\mathrm{E}_{2}$ : estradiol; ECG: electrocardiogram; Endo-PAT: Endothelial Pulse Amplitude Testing; FSH: follicle-stimulating hormone; Hcy: homocysteine; HDL: high-density lipoprotein; LDL: low-density lipoprotein; LH: luteinizing hormone; LnRHI: natural logarithm transformation of Reactive Hyperemia Index; $\ln (\mathrm{Lp}(\mathrm{a}))$ : natural logarithm transformation of lipoprotein A; Lp(a): lipoprotein A; PAT: Peripheral Arterial Tone; PRL: prolactin; RHI: Reactive Hyperemia Index; SBP: systemic blood pressure; T3: triiodothyronine; TG: triglyceride; TSH: thyroid stimulating hormone
\end{abstract}

\section{Background}

The cardiovascular complications in patients with eating disorders, especially anorexia nervosa (AN), have been well described, including sinus bradycardia, a prolonged QT interval, decreased left ventricular wall thickness, alterations of left ventricular morphology and function, and silent pericardial effusion [1,2]. There is evidence that these cardiovascular complications are reversible after patients with AN achieve a normal body mass index (BMI) $[2,3]$.

Endothelial dysfunction contributes to atherogenesis and the development of clinical cardiovascular disease [4]. This can occur even in young children with certain medical conditions [5]. Endothelial pulse amplitude tonometry (Endo-PAT) is a new non-invasive method used to assess peripheral vascular function. It is based on noninvasive Peripheral Arterial Tone (PAT) signal technology that measures endothelium-mediated changes in vascular tone using unique biosensors placed on the fingertips. The changes in arterial tone are elicited by creating a down-stream hyperemic response induced by a standard 5-minute occlusion of the brachial artery. Measurements from the contralateral arm are used to control for concurrent nonendothelial dependent changes in vascular tone. The results are automatically calculated as an index of endothelial function. EndoPAT has been validated in multiple studies, including in adolescent populations [6-8]. Digital vascular dysfunction assessed by Endo-PAT

Correspondence to: Patcharapong Suntharos MD, Department of Pediatric Cardiology, Cleveland Clinic Children's Hospital, 9500 Euclid Avenue, Desk M-41, Cleveland, OH 44195, USA; Tel: (216) 445-1580; E-mail: sunthap@ccf.org

Key words: anorexia nervosa, endothelial function, endothelial pulse amplitude Testing (Endo-PAT)

Received: May 01, 2016; Accepted: May 20, 2016; Published: May 24, 2016 
has a strong correlation with multiple cardiovascular risk factors [9].

The association between endothelial dysfunction and an unfavorable lipid profile has also been described in athletic females who have amenorrhea, eating disorders, and osteoporosis, also known as the female athlete triad. The mechanism for endothelial dysfunction and an impaired lipid profile in this group of patients is believed to be related to hypoestrogenism. However, unlike the direct cardiovascular complications, whether the endothelial dysfunction and impaired lipid profile found in young athletic women with amenorrhea is reversible after resumption of menses is still not known [10].

The purpose of this study was to evaluate endothelial function in patients with AN using Endo-PAT and to correlate findings with the patients' histories, and hormonal levels.

\section{Hypothesis}

Patients with AN have abnormal endothelial function demonstrated by endothelial pulse amplitude tonometry (Endo-PAT).

\section{Methods}

\section{Study population}

All patients between the age of 13 and 21 years who were admitted to the adolescent unit at the Cohen Children's Medical Center of New York for eating disorder management, as well as all ambulatory patients seen in the Division of Adolescent Medicine clinic for eating disorder evaluation and treatment, between June 1, 2012 and May 31, 2013, were screened for eligibility. Those who met the diagnostic criteria for AN by DSM-IV [11] were approached for study participation. Healthy control subjects, similar in age and gender distribution, were screened from all patients who presented to the pediatric cardiology and adolescent medicine clinics during the same time period. Subjects with chronic underlying medical conditions (e.g., congenital or acquired heart disease, inflammatory bowel disease, diabetes mellitus, endocrine disease, renal disease), or current cigarette smoking, alcohol, or illegal drug use were excluded. All subjects aged 18 years or greater, or parents of those who were 17 years or younger, gave informed consent, and minors gave assent, before enrolling in the study.

\section{Demographic, anthropometric and blood pressure measurements}

Baseline demographic data were obtained by history, or abstracted from the medical record, and included: race, ethnicity, height, weight, body mass index (BMI), BMI percentile, Tanner stage, heart rate, and systolic and diastolic blood pressures (SBP and DBP). For the subjects with AN, duration of weight loss, percentage of weight loss and last menstrual period were obtained from the subjects' medical records.

\section{Biochemical analyses}

The following hormones were either measured at the time of the study or abstracted from the medical record if the results were within 3 months of the Endo-PAT procedure: total cholesterol (Chol), highdensity lipoprotein (HDL), low-density lipoprotein (LDL), triglyceride (TG), lipoprotein A (Lp(a)), homocysteine (Hcy), thyroid stimulating hormone (TSH), triiodothyronine (T3), follicle-stimulating hormone (FSH), luteinizing hormone (LH), estradiol $\left(\mathrm{E}_{2}\right)$, and prolactin (PRL). All biochemical analyses were performed at the Northwell Health Core Laboratory.

\section{Electrocardiogram}

Electrocardiograms (ECGs) performed within one week prior to enrollment to the study were obtained from the medical record. For subjects who did not have an ECG performed within that timeframe, an ECG was performed in the pediatric cardiology clinic. All ECGs were interpreted by a pediatric cardiologist.

\section{Peripheral arterial tonometry}

Peripheral arterial tonometry testing was performed in a quiet, restful environment with a comfortable temperature (between $72^{\circ}$ to $75^{\circ} \mathrm{F}$ ). The subjects were asked to fast at least 4 hours prior to the procedure and to avoid caffeine consumption for 12 hours before testing. Patients who were on psychotropic medications were not excluded from the study. The testing protocol was performed using the Endo-PAT device as instructed by the manufacturer (Itamar Medical, Caesarea, Israel). Endo-PAT is a Federal Drug Administration (FDA)approved commercially available tool introduced by the Itamar Corporation. This machine non-invasively measures peripheral arterial tone (PAT), which reflects changes in arterial pulsatile blood volume. The PAT amplitude is equivalent to the arterial pulse volume. The device comprises a pneumatic plethysograph that applies uniform pressure to the entire distal finger surface, allowing measurement of pulse volume changes in the finger. Non-invasive pneumatic probes were placed on the index fingers of both hands. The pulse wave amplitude was recorded continuously from both index fingers. A reactive hyperemia procedure was performed by occluding the brachial artery of the nondominant arm with a blood pressure cuff at $250 \mathrm{mmHg}$ for 5 minutes. The tracing in the non-occluded arm served as a control for changes in overall physiologic state. The Endo-PAT data were analyzed by a computerized, automated algorithm (Itamar Medical), without any input from the examiner. Endo-PAT provides an index of endothelial function in two forms: The Reactive Hyperemia Index (RHI) and the natural log transformation of RHI (LnRHI). The RHI was defined as the ratio of the average pulse amplitude during the 1 minute period beginning after exactly 90 seconds of reactive hyperemia compared with the average pulse amplitude during the pre-occlusion baseline period. The LnRHI is a similar index to the RHI after natural log transformation. The LnRHI provides a better double-sided distribution that is closer to normal distribution than RHI. It offers better separation between disease states.

\section{Statistical analysis}

Demographic parameters were compared between groups using the two-sample t-test for continuous parameters and the chi-square test, or Fisher's Exact test, for categorical parameters.

The two-sample t-test was used to compare outcomes of interest between the subjects with AN and the healthy, normal controls. Not all outcome variables met the standard assumptions of normality and equality of variance required to conduct a t-test. However, the data was also analyzed using the Mann-Whitney $U$ test, a non-parametric counterpart to the t-test. Results between the t-test and the MannWhitney U test did not differ qualitatively. Therefore, for ease of interpretation and consistency, only results from the t-test are reported and presented here. If results between parametric and non-parametric tests differed qualitatively, then an appropriate transformation was applied, such as the natural logarithm, in order to meet the necessary assumptions of the t-test (this applied specifically to $\mathrm{Lp}(\mathrm{a})$ ).

The correlation of demographic and laboratory results with the EndoPAT findings was examined using the Spearman correlation coefficient. 
All statistical analyses were conducted in SAS version 9.3 (SAS Institute, Cary, NC). Results were considered significant at a p-value of less than 0.05 for all primary aim which was to compare RHI and LnRHI between two groups. A Bonferroni-like adjustment, alpha level of 0.01 , was applied to all secondary aims which were the comparison of other biochemical parameters and the correlation of demographic and laboratory results with EndoPAT results.

The study protocol was reviewed and approved by the Northwell Health Institutional Review Board.

\section{Results}

During the study period, 254 subjects who came for eating disorder management, either as an in-patient admission or in the ambulatory clinic, were screened for eligibility. A total of 35 (13.78\%) subjects were eligible, $27(77.14 \%)$ of whom were consented and enrolled for the study. The Endo-PAT was performed on 21 (77.78\%) subjects; $5(18.52 \%)$ subjects did not come for the appointment and $1(3.7 \%)$ subject withdrew from the study.

For the control group, 350 subjects were screened and 37 (10.57\%) subjects were eligible for the study. Twenty-four (64.86\%) subjects consented, $2(8.33 \%)$ subjects did not come for the appointment, 1 (4.17\%) subject withdrew from the study, and 2 (8.33\%) subjects were excluded from the analysis because of lack of cooperation during the Endo-PAT study, which resulted in significant movement artifacts in one subject and a poor Endo-PAT signal in the other subject.

Of the total of 40 subjects who had the Endo-PAT performed (21 patients with $\mathrm{AN}(52.5 \%)$ and 19 normal, healthy controls (47.5\%)), two subjects (5\%) (1 patient with AN and 1 healthy control) were male. All of the patients with AN were non-Hispanic White. In the control group, 1 (5.26\%) subject was Asian, 4 (21.05\%) were African American, $1(5.26 \%)$ was Hispanic White, and the remaining were non-Hispanic White (68.43\%). A description of demographic parameters is shown in Table 1.

There were no significant differences between the RHI of $1.84 \pm$ 0.51 in the $\mathrm{AN}$ group and $1.90 \pm 0.69$ in the control group $(\mathrm{P}=0.7542)$ or LnRHI of $0.58 \pm 0.29$ and $0.58 \pm 0.35(\mathrm{P}=0.9497)$ respectively.

The patients with AN had a lower mean weight $(\mathrm{P}<0.0001)$, height $(\mathrm{P}=0.0207)$, BMI $(\mathrm{P}<0.0001)$, resting HR $(\mathrm{P}<0.0001)$, SBP $(\mathrm{P}<0.0001)$

Table 1. Demographic information.

\begin{tabular}{|l|c|c|c|}
\hline Variable & Anorexia Nervosa & Controls & P value \\
\hline Total patients $(\mathrm{N})$ & 21 & 19 & \\
\hline Age (years) & $16.44 \pm 1.50$ & $16.87 \pm 2.13$ & 0.4579 \\
\hline Height $(\mathrm{cm})$ & $160.11 \pm 6.38$ & $165.02 \pm 6.48$ & 0.0207 \\
\hline Weight $(\mathrm{Kg})$ & $41.14 \pm 6.73$ & $60.86 \pm 9.62$ & $<0.0001$ \\
\hline Body Mass Index $\left(\mathrm{Kg} / \mathrm{m}^{2}\right)$ & $16.00 \pm 2.12$ & $22.29 \pm 2.74$ & $<0.0001$ \\
\hline Weight loss (\%) & $23.06 \pm 8.04$ & $\mathrm{n} / \mathrm{a}$ & \\
\hline Weight loss Duration (months) & $13.71 \pm 12.10$ & $\mathrm{n} / \mathrm{a}$ & \\
\hline Resting heart rate (BPM) & $52.57 \pm 10.91$ & $67.26 \pm 7.38$ & $<0.0001$ \\
\hline SBP (mmHg) & $95.24 \pm 8.83$ & $111.79 \pm 9.23$ & $<0.0001$ \\
\hline DBP (mmHg) & $58.00 \pm 7.22$ & $64.26 \pm 8.18$ & 0.0141 \\
\hline Tanner Stage & & & 0.9608 \\
\hline \multicolumn{1}{|c|}{3} & $5(23.81)$ & $5(26.32)$ & \\
\hline \multicolumn{1}{|c|}{4} & $13(61.90)$ & $11(57.89)$ & \\
\hline \multicolumn{1}{|c|}{5} & $3(14.29)$ & $3(15.79)$ & \\
\hline
\end{tabular}

Value are expressed as mean $\pm \mathrm{SD}$ or number of patients (percentage). $\mathrm{BPM}=$ beats per minute, $\mathrm{SBP}=$ systolic blood pressure, $\mathrm{DBP}=$ diastolic blood pressure. $\mathrm{P}$-value of less than 0.05 is considered statistically significant. and DBP $(\mathrm{P}=0.0141)$ than the healthy normal subjects. No significant differences were observed in mean age $(\mathrm{P}=0.4579)$ or Tanner stage $(\mathrm{P}=0.9608)$ between the groups.

The patients with AN also had a lower mean HR during Endo-PAT testing $(\mathrm{P}<0.0001), \mathrm{T} 3(\mathrm{P}<0.0001)$, $\mathrm{LH}(\mathrm{P}=0.0055)$ and $\mathrm{E}_{2}(\mathrm{P}=0.0052)$ as compared to the healthy, normal controls (Table 2). The Chol level was higher in the AN subjects compared to the healthy controls $(\mathrm{P}=0.0004)$. There were no significant differences between the groups for HDL $(\mathrm{P}=0.0546), \mathrm{LDL}(\mathrm{P}=0.0108), \mathrm{TG}(\mathrm{P}=0.3182), \ln (\mathrm{Lp}(\mathrm{a}))(\mathrm{P}=0.0447)$, Hcy $(\mathrm{P}=0.6833)$, TSH $(\mathrm{P}=0.4421)$, FSH $(\mathrm{P}=0.6182)$ or $\mathrm{PRL}(\mathrm{P}=0.1052)$ at a significance level of 0.01 .

We further examined the correlation of demographic and laboratory values with Endo-PAT finding using the Spearman correlation coefficient (Table 3). No correlations were observed between BMI, HR during EndoPAT, weight loss, duration of amenorrhea, Chol,

Table 2. Comparison of heart rate and biochemical analysis data

\begin{tabular}{|l|c|c|c|c|c|}
\hline Variable & \multicolumn{2}{|c|}{ Anorexia Nervosa } & \multicolumn{2}{c|}{ Controls } & P value \\
\hline & $\mathrm{N}$ & & $\mathrm{N}$ & & \\
\hline $\begin{array}{l}\text { HR during EndoPAT } \\
\text { testing (BPM) }\end{array}$ & 21 & $53.57 \pm 7.88$ & 19 & $67.79 \pm 8.89$ & $<0.0001$ \\
\hline Chol (mg/dL) & 13 & $181.77 \pm 22.55$ & 6 & $138.83 \pm 10.46$ & 0.0004 \\
\hline HDL (mg/dL) & 13 & $71.23 \pm 15.36$ & 6 & $57.00 \pm 9.86$ & 0.0546 \\
\hline LDL (mg/dL) & 13 & $95.62 \pm 19.81$ & 6 & $69.67 \pm 14.31$ & 0.0108 \\
\hline Triglyceride (mg/dL) & 13 & $78.08 \pm 28.19$ & 6 & $61.33 \pm 42.36$ & 0.3182 \\
\hline In(Lipoprotein A) & 11 & $3.46 \pm 0.85$ & 5 & $4.37 \pm 0.51$ & 0.0447 \\
\hline Homocysteine $(\mu \mathrm{mol} / \mathrm{L})$ & 11 & $10.43 \pm 2.58$ & 6 & $9.90 \pm 2.33$ & 0.6833 \\
\hline Triiodothyronine $(\mathrm{ng} / \mathrm{dL})$ & 13 & $67.46 \pm 19.68$ & 6 & $110.33 \pm 10.52$ & $<0.0001$ \\
\hline TSH (IU/mL) & 21 & $2.59 \pm 1.93$ & 6 & $1.94 \pm 1.27$ & 0.4421 \\
\hline FSH (IU/mL) & 20 & $4.20 \pm 3.62$ & 5 & $5.06 \pm 2.30$ & 0.6182 \\
\hline LH (IU/mL) & 20 & $1.30 \pm 2.00$ & 5 & $4.80 \pm 3.32$ & 0.0055 \\
\hline Estradiol $(\mathrm{pg} / \mathrm{mL})$ & 18 & $16.56 \pm 10.07$ & 5 & $140.60 \pm 178.99$ & 0.0052 \\
\hline Prolactin $(\mathrm{ng} / \mathrm{mL})$ & 19 & $8.45 \pm 5.02$ & 5 & $13.34 \pm 8.31$ & 0.1052 \\
\hline
\end{tabular}

Value are expressed as mean $\pm \mathrm{SD} . \mathrm{HR}=$ heart rate, $\mathrm{BPM}=$ beats per minute, $\mathrm{Chol}=$ total cholesterol, HDL = high-density lipoprotein, $\mathrm{LDL}=$ low-density lipoprotein, $\mathrm{TSH}=$ thyroid stimulating hormone, $\mathrm{FSH}=$ follicle-stimulating hormone, $\mathrm{LH}=$ luteinizing hormone. $\mathrm{P}$-value of less than 0.01 is considered statistically significant.

Table 3. Correlation between demographic and biochemical parameters with EndoPAT results.

\begin{tabular}{|l|c|c|c|}
\hline Parameter & $\mathbf{N}$ & Correlation coefficient (p) & P-value \\
\hline Body Mass Index & 40 & 0.2196 & 0.1734 \\
\hline HR during EndoPAT testing & 40 & -0.1688 & 0.2978 \\
\hline Weight Loss $^{1}$ & 21 & -0.4139 & 0.0621 \\
\hline Amenorrhea duration $^{1,2}$ & 18 & -0.2345 & 0.3489 \\
\hline Total cholesterol & 19 & 0.0649 & 0.7918 \\
\hline HDL & 19 & -0.3423 & 0.1515 \\
\hline LDL & 19 & 0.1845 & 0.4497 \\
\hline Triglyceride & 19 & 0.5266 & 0.0206 \\
\hline In(Lipoprotein A) & 16 & -0.0412 & 0.8797 \\
\hline Homocysteine & 17 & 0.5841 & 0.0138 \\
\hline Triiodothyronine & 19 & -0.1984 & 0.4155 \\
\hline TSH & 27 & -0.1112 & 0.5810 \\
\hline FSH & 25 & 0.1444 & 0.4910 \\
\hline LH & 25 & -0.0995 & 0.6361 \\
\hline Estradiol & 23 & -0.1950 & 0.3726 \\
\hline Prolactin & 24 & -0.3480 & 0.0957 \\
\hline
\end{tabular}

$\mathrm{N}=$ total patients, $\mathrm{HR}=$ heart rate, $\mathrm{HDL}=$ high-density lipoprotein, $\mathrm{LDL}=$ low-density lipoprotein, $\mathrm{TSH}=$ thyroid stimulating hormone, $\mathrm{FSH}=$ follicle-stimulating hormone, $\mathrm{LH}=$ luteinizing hormone. P-value of less than 0.01 is considered statistically significant. ${ }^{1}$ Only applies to anorexic subjects.

${ }^{2}$ Two subjects were primary amenorrhea and one subject was male. 
HDL, LDL, TG, In(Lp(a)), Hcy, TSH, T3, FSH, LH, E, PRL and RHI at a significance level of 0.01 . No significant abnormalities were found on ECG in the patients with AN except for various degrees of sinus bradycardia.

\section{Discussion}

To our knowledge, this is the first report that examines endothelial function in adolescent patients with AN by studying the relationship of baseline digital pulse amplitude and digital pulse amplitude hyperemic response, a non-invasive measure of vascular endothelial function. We observed no significant differences in both RHI and LnRHI between the AN subjects and the control group. In addition, we found no significant correlations between RHI and BMI, HR, weight loss, duration of amenorrhea, Chol, HDL, LDL, TG, In(Lp(a)), Hcy, TSH, T3, FSH, LH, $\mathrm{E}_{2}$, and PRL. We found significantly lower HR, T3, LH and $\mathrm{E}_{2}$ level in the AN group compared to controls. We also found that total cholesterol was significantly higher in the AN group. These findings confirm previous published studies [10,12-16].

There is strong evidence to support that $\mathrm{T}_{3}$ and $\mathrm{E}_{2}$ can potentially affect endothelium-dependent vasodilatation [17-22]. In various studies, it has been shown that endothelial function changes during puberty in both human $[23,24]$ and animal models $[25,26]$. Adolescents in the advanced stages of pubertal development have a higher peripheral vasodilatory response, as measured by EndoPAT, compared to the initial stages of pubertal development $[23,24]$. Bhangoo et al., [23] studied 89 children and adolescents at different stages of puberty, which was based on ultrasensitive estrogen assays, and found significant positive correlations between the RHI and steroid hormone levels. These findings are similar to data in the adult population which showed that estrogens enhance the endothelialdependent flow-mediated vasodilatation [18-20] Estrogens exert their effects by binding to the estrogen receptor (ER) located on the vascular endothelial and smooth muscle cells of the blood vessels [21,22].

Napoli et al. [17] conducted a double blind, placebo-controlled trial to study the effect of acute changes in serum T3 concentration on endothelial function in 10 healthy subjects aged $24 \pm 1$ year. They found that T3 exerts direct and significant acute effects on blood vessel resistance by enhancing endothelial dilatation, with a significant increase in forearm blood flow in the extremity receiving a continuous $\mathrm{T}_{3}$ infusion when compared to the placebo.

Another interesting finding in our study was a significant higher Chol and the tendency of higher LDL levels in patients with AN compared to controls $(\mathrm{P}=0.0108)$. An increase in the plasma levels of Chol, especially LDL, is a well-known risk factor for atherosclerotic disease [27]. Previous studies have demonstrated that decreased levels of endogenous estrogen unfavorably modify the lipid profile and vascular function in postmenopausal women [28-30]. Elevated lipid levels, especially LDL, have also been reported in premenopausal women with hypoestrogenic conditions due to caloric deficiency such as in AN or athletic amenorrhea $[10,31,32]$. There are several reasons for this abnormal lipid profile, which include changes in thyroid hormones, increased lipolysis and decreased endogenous cholesterol synthesis with a resulting decrease in LDL removal [33].

In contrast to previously published studies, we failed to show any correlations between $\mathrm{E}_{2}, \mathrm{~T} 3$, Chol and LDL with RHI. In addition to a small sample size, this may be because AN patients, despite having well established laboratory risk factors for arteriosclerosis, have normal endothelial function. We suggest that future studies be performed to investigate whether our findings in patients with $\mathrm{AN}$ are reproducible in vitro, such as by biochemical markers of endothelial function, to confirm our results. Furthermore, prospective, longitudinal studies to determine whether patients with AN have a higher incidence of arteriosclerosis or acute coronary syndrome in adulthood, as long term consequences of these abnormal biomarkers at a very young age, should be considered.

\section{Limitations}

One limitation of this study is that there were some subjects who had missing laboratory data, which could result in limiting our ability to show significant correlations between the RHI and other variables. The norm for RHI and LnRHI values in pediatric populations have not been determined, which also limited our ability to evaluate whether some of the patients with AN have abnormal endothelial function and to understand the factors that might have caused the abnormality. A further study with a larger cohort, including age-, gender- and Tanner stage-matched controls, is warranted.

\section{Conclusion}

In our cohort, there were no significant differences in RHI or LnRHI between a group of patients with $\mathrm{AN}$ and controls. There was a significantly increased mean $\mathrm{Chol}$ and decreased $\mathrm{HR}, \mathrm{T}_{3}, \mathrm{LH}$ and $\mathrm{E}_{2}$ levels in the patients with AN compared to controls, which confirms other published data. No correlations were observed between RHI and BMI, HR, weight loss, duration of amenorrhea, Chol, HDL, LDL, TG, In(Lp(a)), Hcy, TSH, T3, FSH, LH, E, and PRL.

\section{Funding source}

Cohen Children's Medical Center, Northwell Health Pediatric Cardiology Research Fund. No external funding.

\section{Financial Disclosure}

The authors declare no financial relationships relevant to this article to disclose.

\section{Conflict of interest}

The authors declare no conflicts of interest relevant to this article to disclose.

\section{References}

1. Katzman DK (2005) Medical complications in adolescents with anorexia nervosa: review of the literature. Int J Eat Disord 37 Suppl: S52-59.[Crossref]

2. DiVasta AD, Walls CE, Feldman HA, Quach AE, Woods ER, et al. (2010) Malnutrition and hemodynamic status in adolescents hospitalized for anorexia nervosa. Arch Pediatr Adolesc Med 164: 706-713.[Crossref]

3. Mont L, Castro J, Herreros B, Paré C, Azqueta M, et al. (2003) Reversibility of cardiac abnormalities in adolescents with anorexia nervosa after weight recovery. J Am Acad Child Adolesc Psychiatry 42: 808-813.[Crossref]

4. Widlansky ME, Gokce N, Keaney JF Jr, Vita JA (2003) The clinical implications of endothelial dysfunction. J Am Coll Cardiol 42: 1149-1160.[Crossref]

5. Celermajer DS, Sorensen KE, Gooch VM, Spiegelhalter DJ, Miller OI, et al. (1992) Non-invasive detection of endothelial dysfunction in children and adults at risk of atherosclerosis. Lancet 340: 1111-1115.[Crossref]

6. Selamet Tierney ES, Newburger JW, Gauvreau K, Geva J, Coogan E, et al. (2009) Endothelial pulse amplitude testing: feasibility and reproducibility in adolescents. $J$ Pediatr 154: 901-905.[Crossref]

7. Bonetti PO, Pumper GM, Higano ST, Holmes DR, Kuvin, JT, et al. (2004) Noninvasive identification of patients with early coronary atherosclerosis by assessment of digital reactive hyperemia. J Am Coll Cardiol 44: 2137-41. 
8. Hamburg NM, Benjamin EJ (2009) Assessment of endothelial function using digital pulse amplitude tonometry. Trends Cardiovasc Med 19: 6-11.[Crossref]

9. Hamburg NM, Keyes MJ, Larson MG, Vasan RS, Schnabel R, et al. (2008) Crosssectional relations of digital vascular function to cardiovascular risk factors in the Framingham Heart Study. Circulation 117: 2467-2474.[Crossref]

10. Rickenlund A, Eriksson MJ, Schenck-Gustafsson K, Hirschberg AL (2005) Amenorrhea in female athletes is associated with endothelial dysfunction and unfavorable lipid profile. J Clin Endocrinol Metab 90:1354-59.

11. American Psychiatric Association. Diagnostic and Statistical Manual Disorders, 4th Ed, Text Revision, Washington, DC 2000, 589.

12. Tamai H, Mori K, Matsubayashi S (1986) Hypothalamic-pituitary-thyroidal dysfunctions in anorexia nervosa. Psychother Psychosom 46:127-31.

13. Leslie RD, Isaacs AJ, Gomez J, Raggatt PR, Bayliss R (1978) Hypothalamo-pituitarythyroid function in anorexia nervosa: influence of weight gain. $\operatorname{Br}$ Med J 2: 526-528. [Crossref]

14. Becker AE, Grinspoon SK, Klibanski A, Herzog DB (1999) Eating disorders. N Engl J Med 340: 1092-1098.[Crossref]

15. Croxson MS, Ibbertson HK (1977) Low serum triiodothyronine (T3) and hypothyroidism in anorexia nervosa. J Clin Endocrinol Metab 44: 167-174.[Crossref]

16. Misra M, Miller KK, Tsai P, Stewart V, End A, et al. (2006) Uncoupling of cardiovascular risk markers in adolescent girls with anorexia nervosa. J Pediatr 149: 763-769.[Crossref]

17. Napoli R, Guardasole V, Angelini V, Zarra E, Terracciano D, et al. (2007) Acute effects of triiodothyronine on endothelial function in human subjects. $J$ Clin Endocrinol Metab 92: 250-254.[Crossref]

18. Lieberman EH, Gerhard MD, Uehata A, Walsh BW, Selwyn AP, et al. (1994) Estrogen improves endothelium-dependent, flow-mediated vasodilation in postmenopausal women. Ann Intern Med 121: 936-941.[Crossref]

19. Williams JK, Adams MR, Klopfenstein HS (1990) Estrogen modulates responses of atherosclerotic coronary arteries. Circulation 81: 1680-1687.[Crossref]

20. Gisclard V, Miller VM, Vanhoutte PM (1988) Effect of 17 beta-estradiol on endothelium-dependent responses in the rabbit. J Pharmacol Exp Ther 244: 19-22. [Crossref]
21. Mendelsohn ME, Karas RH (1994) Estrogen and the blood vessel wall. Curr Opin Cardiol 9: 619-626.[Crossref]

22. Farhat MY, Lavigne MC, Ramwell PW (1996) The vascular protective effects of estrogen. FASEB J 10: 615-624.[Crossref]

23. Bhangoo A, Sinha S, Rosenbaum M, Shelov S, Ten S (2011) Endothelial function as measured by peripheral arterial tonometry increases during pubertal advancement Horm Res Paediatr 76: 226-233.[Crossref]

24. Radtke T, Khattab K, Eser P, Kriemler S, Saner H, et al. (2012) Puberty and microvascular function in healthy children and adolescents. J Pediatr 161: 887-891. [Crossref]

25. Chatrath R, Ronningen KL, LaBreche P, Severson SR, Jayachandran M, et al. (2003) Effect of puberty on coronary arteries from female pigs. J Appl Physiol 95: 1672-1680. [Crossref]

26. Chatrath R, Ronningen KL, Severson SR, LaBreche P, Jayachandran M, et al. (2003) Endothelium-dependent responses in coronary arteries are changed with puberty in male pigs. Am J Physiol Heart Circ Physiol 285: H1168-1176.[Crossref]

27. Brown MS, Goldstein JL (1986) A receptor-mediated pathway for cholesterol homeostasis. Science 232: 34-47.[Crossref]

28. Cid MC, Schnaper HW, Kleinman HK (2002) Estrogens and the vascular endothelium. Ann N Y Acad Sci 966: 143-157.[Crossref]

29. Mendelsohn ME1 (2002) Protective effects of estrogen on the cardiovascular system Am J Cardiol 89: 12E-17E.[Crossref]

30. Kannel WB, Hjortland MC, McNamara PM, Gordon T (1976) Menopause and risk of cardiovascular disease: the Framingham study. Ann Intern Med 85: 447-452.[Crossref]

31. Case T, Lemieux S, Kennedy SH, Lewis GF (1999) Elevated plasma lipids in patients with binge eating disorders are found only in those who are anorexic. Int J Eat Disord 25: 187-193.[Crossref]

32. Feillet F, Feillet-Coudray C, Bard JM, Parra HJ, Favre E, et al. (2000) Plasma cholesterol and endogenous cholesterol synthesis during refeeding in anorexia nervosa. Clin Chim Acta 294: 45-56.[Crossref]

33. Weinbrenner T, Züger M, Jacoby GE, HerpertzS, Liedtke R, et al. (2004) Lipoprotein metabolism in patients with anorexia nervosa: a case-control study investigating the mechanisms leading to hypercholesterolaemia. Br J Nutr 91: 959-969.

Copyright: $₫ 2016$ Suntharos P. This is an open-access article distributed under the terms of the Creative Commons Attribution License, which permits unrestricted use, distribution, and reproduction in any medium, provided the original author and source are credited. 